Revista de Psicología Vol. 34 (2), 2016 (ISSN 0254-9247)

\title{
Estilos relacionales parentales: estudio con adolescentes y sus padres
}

\author{
Alvaro Capano Bosch ${ }^{1}$, María del Luján González Tornaría ${ }^{2}$ \\ y Natalie Massonnier ${ }^{3}$ \\ Universidad Católica del Uruguay
}

Se estudiaron los estilos parentales según la percepción de padres, madres y sus hijos e hijas de 12 ańos. Se aplicaron las escalas de Afecto y de Normas y Exigencias a 107 padres y madres y 107 hijos e hijas. Se encontraron diferencias significativas en la percepción de padres y madres, en el factor Crítica/Rechazo y en la subescala Inductiva, según el sexo de los hijos que respondieron. Las percepciones de hijos e hijas arrojaron diferencias significativas para los padres, en las escalas Afecto e Indulgente y para las madres en las escalas Afecto, Indulgente e Inductiva. Los padres con nivel de enseñanza primaria aparecieron significativamente más inductivos que quienes tuvieron estudios universitarios.

Palabras clave: familia, estrategias de socialización, estilos parentales, adolescencia.

\section{Parental relational styles: A study with adolescents and their parents}

Perceptions of parenting styles by parents and their 12-year-old preadolescents are studied One hundred seven parents and their adolescents enrolled in the first year of secondary education in one of four Catholic schools in Montevideo completed Affection, Rules and Requirements questionnaires. There were significant differences in parent perception in the critical / rejection and inductive factor subscale, depending on the gender of the adolescent. Preadolescent perceptions exhibit significant differences in indulgent and affection scales for father, and in affection, indulgent and inductive scales for mothers. Parents with a primary school education level appear significantly more inductive than those who were are university graduates.

Key words: Family, socialization strategies, parenting styles, preadolescence.

1 Maestrando en Psicología Clínica Familiar Sistémica e investigador asociado al Departamento de Psicología del Desarrollo y Educacional de la Universidad Católica del Uruguay UCU CP. 11600. Dirección postal: 8 de Octubre 2738. 11600 Montevideo, Uruguay. Contacto: alvarocapano@gmail.com

2 Doctora en Ciencias de la Educación y docente en la Universidad Católica del Uruguay. UCU CP. 11600. Dirección postal: 8 de Octubre 2738. 11600 Montevideo, Uruguay. Contacto: mgonzale@ucu.edu.uy

3 Licenciada en Psicología y profesora en la Universidad Católica del Uruguay. UCU CP. 11600. Dirección postal: 8 de Octubre 2738. 11600 Montevideo, Uruguay. Contacto: natalie.massonnier@gmail.com 


\section{Estilos parentais relacionais: Um estudo em adolescentes e seus pais}

No artigo são estudadas as percepçóes dos pais, mais e seus filhos e filhas menores de 12 anos, sobre os estilos parentais em suas famílias. Foram aplicadas as escalas de Afeto, Normas e Exigências a 107 pais e mais e 107 adolescentes. Os resultados mostram diferenças significativas nas percepções sobre os pais e mais nas dimensões de crítica/rejeição e indutiva, dependendo se os respondentes são homens ou mulheres. As percepçôes de filhos e filhas sobre os pais mostraram diferenças significativas nas escalas de afeto e indulgente e as percepçóes de filhos e filhas sobre as mais mostraram diferenças nas escalas de afeto, indulgente e indutiva. Os pais com nível de educaçáo primaria apareceram como mais indutivos que os pais que estudaram na universidade.

Palavras-chave: família, estratégias de socialização, estilos parentais, adolescência. 
Las familias presentan actualmente una multiplicidad de realidades en relación a sus estructuras, límites, funciones y roles de sus integrantes (Gimeno, 2007). En las últimas décadas, han tenido que vivir y sobrellevar profundas trasformaciones, para algunos autores, una auténtica revolución familiar (Valdivia, 2007). Las familias en tanto sistemas están constituidas por una red de relaciones, cumplen con las necesidades biológicas y psicológicas para la supervivencia y conservación de la especie humana, son un pilar trascendente en la vida psíquica de las personas (Ceberio, 2013). Son responsables por la estabilidad emocional de sus integrantes, tanto en la nińez como en la adultez (Flaquer, 1998). No existe otra instancia social que logre reemplazarlas en la satisfacción de las necesidades psicoafectivas de todos sus integrantes y su retroalimentación emocional (Hernández, 1997).

Así concebidas, las familias se encargan de socializar a sus nuevos integrantes, y constituyen el lugar donde se adquiere el lenguaje, los esquemas básicos para interpretar la realidad, una visión y un sentido de la vida y también de sí mismo (Gervilla, 2008). Es en su seno que se alcanzan las habilidades cognitivas, emocionales y sociales necesarias para el funcionamiento en la sociedad (Cortés, Cantón \& Cantón Cortés, 2011) y donde el ser humano permanece un largo tiempo en momentos cruciales de su desarrollo evolutivo como el neonatal, la infancia y la adolescencia (Nardone, Giannotti \& Rocchi, 2003). Es además el principal vehículo en la transmisión cultural, donde se producen y desarrollan los estímulos relacionales de mayor relevancia para la maduración individual, según Linares (2009), la nutrición relacional.

La Teoría General de Sistemas (Bertalanffy, 1968), se refiere a la familia como un sistema abierto que se encuentra constituido por un conjunto de elementos en interacción dinámica. Estas interacciones que se ejercen dentro de la familia deben ser entendidas como parte de un sistema dinámico y complejo, con intercambios de información y regulaciones entre las partes integrantes del sistema (Oliva \& Arranz, 2011). 
Las personas reciben así presiones intrafamiliares y extrafamiliares que requieren una transformación constante de sus miembros a lo largo del ciclo vital con el objetivo de crecer y favorecer su continuidad (Minuchin, 1974). Desde hace tiempo se entiende que el proceso de interacción familiar es bidireccional, ya que también los padres son socializados por los hijos: muy probablemente se lo pueda considerar un proceso con características circulares, más acentuado en la medida que los hijos crecen (Musitu \& Cava, 2001).

Los actuales modelos teóricos sobre socialización familiar son de mayor complejidad que los unidireccionales: además de estudiar las interacciones y su bidireccionalidad, son ecológicos al tener en cuenta los contextos en que se dan dichas interacciones, contemplan la influencia genética y reconocen la posibilidad de intervenir y educar a las familias, justamente por considerarlas sistemas abiertos (Oliva \&Arranz, 2011).

Desde hace varias décadas el concepto de estilo parental acuñado por Baumrind (1966), ha sido ampliamente utilizado en investigaciones acerca de las consecuencias de la socialización familiar sobre el desarrollo de niños y adolescentes. Se habla de estilos parentales, cuando se presenta un conglomerado de acciones parentales hacia los hijos que les son comunicadas a estos, y que, actuando conjuntamente, crean un clima emocional en el que se manifiestan las conductas de los padres (Darling \& Steinberg, 1993). El estilo educativo parental es el contexto emocional donde toman relevancia las conductas educativas específicas (González Tornaría, Vandemeulebroecke \& Colpin, 2001). Se habla de estilo por su permanencia y estabilidad a lo largo del tiempo, aunque pueden existir modificaciones (Climent, 2009), ya que los padres escogen dentro de un marco amplio y flexible los posibles modelos educativos (Ceballos \& Rodrigo, 1998).

Dentro de la tipología de estilos se encuentran el "modelo de autoridad parental" de Baumrind (1966) y el "modelo de Maccoby y Martín (1983)". Baumrind (1966), propone tres tipos de estilos parentales en función del grado de control que los padres ejercen sobre sus hijos: el estilo autoritario, el permisivo y el democrático. Aportes posteriores como los de Maccoby y Martin (1983), han servido para reformular 
la propuesta hecha por Baumrind (1966), proponiendo cuatro estilos parentales a partir de dos dimensiones afecto/comunicación y control/ establecimiento de límites. El apoyo/afecto refiere al amor, a la aprobación, a la aceptación y a la ayuda que se les brinda a los hijos. La dimensión control parental hace referencia al disciplinamiento que intentan conseguir los padres, de esta manera los padres controlan y/o supervisan el comportamiento de sus hijos. A partir de estas dimensiones se desarrollan cuatro estilos parentales, el autoritario, el permisivo, el democrático y el negligente. A los efectos del presente estudio, nos centraremos en la propuesta hecha por Baumrind (1966).

\section{Estilo democrático}

Los padres que ejercen el estilo democrático (afecto, control y exigencia de madurez) tienen hijos con un mejor ajuste emocional y comportamental (Oliva, Parra, Sánchez-Queija \& López, 2007). Son padres que estimulan la expresión de las necesidades de sus hijos, promueven la responsabilidad y otorgan autonomía (Torío, Peña \& Inda, 2008), logran en estos una mejor adaptación y una menor propensión a experimentar ira o frustración o a manifestar problemas como la agresión (Eisenberg et. al, 2005). Este estilo tiene un impacto muy positivo en el desarrollo psicológico de los hijos (Valdivia, 2010), quienes manifiestan un estado emocional estable y alegre, una elevada autoestima y autocontrol (Arranz, Bellido, Manzano, Martín \& Olabarrieta, 2004), un nivel superior de razonamiento moral y menos conductas externalizantes (Bornstein \& Zlotnik, 2008) e internalizantes (Carpio, García-Linares, de la Torre, Cerezo \& Casanova, 2014), así como menor nivel de estrés cotidiano (García-Linares, de la Torre, Carpio, Cerezo \& Casanova, 2014), principalmente cuando ambos padres se manejan desde este estilo (Cortés, Cantón \& Cantón Cortés, 2011; Oliva, Parra \& Arranz, 2008; Simons \& Conger, 2007; Steinberg, 2001). El estilo democrático se asocia a una mayor autoestima, un mejor rendimiento académico (Maccoby \& Martin, 1983; Sabán, Herruzo \& Raya, 2013), mayor desarrollo moral y social (López-Soler, Puerto, López-Pina \& Prieto, 2009) mayor probabilidad de ser sociables (Alonso \& Román, 2005; Lila \& Gracia, 2005), 
una menor impulsividad, una mayor intención de alcanzar metas positivas, menor prevalencia de experiencia sexual y mayor rechazo de diversas formas de sexismo (Osorio de Rebellón, Rivas, Estévez, Calatrava \& López del Burgo, 2009), menor conflictiva con sus padres y mayor bienestar psicológico (Arranz, Bellido et al., 2004; Torío, Peńa \& Rodríguez, 2008), mejor ajuste conductual (Oliva, Parra, Sánchez-Queija \& López, 2007; Parra, Oliva \& Sánchez-Queija, 2004) menor riesgo de consumo de tabaco en adolescentes (Cano, Solanas, Marí-Klose \& Marí-Klose, 2012), menor riesgo de abuso de drogas en general (Becońa, Martínez, Calafat, Fernández Hermida \& Secades-Villa, 2012).

\section{Estilo permisivo}

Los niños y adolescentes que viven en hogares con estilo permisivo demuestran ser poco obedientes, tienen dificultad en la interiorización de valores, viven situaciones de agresividad en la familia, tienen baja autoestima, padecen de falta de confianza, tienen bajos niveles de control de sus impulsos, así como mayor riesgo de consumo de drogas y alcohol (Torío, Peña \& Inda, 2008). No obstante, algunos autores refieren en sus estudios que el estilo parental permisivo funciona como factor de prevención del consumo de sustancias y es el estilo que presenta un menor consumo (Fuentes, Alarcón, García \& Gracia, 2014; Martínez, Fuentes, García \& Madrid 2013). Cabe aclarar que los problemas de conducta que presentan los adolescentes con padres permisivos son menores que los que presentan los adolescentes con padres autoritarios y negligentes (Gracia, Fuentes \& García, 2010). Los padres permisivos les conceden la posibilidad de tomar decisiones que no les competen. Son padres con miedo a influir demasiado en sus hijos, con temor a veces a sus reacciones, no sabiendo relacionarse con ellos, se sienten culpables por el escaso tiempo que les dispensan (Valdivia, 2010), les plantean pocas exigencias, confían solo en la razón, siendo los niños quienes regulan sus actividades (Cortés et al., 2011). Los padres promueven una importante autonomía en sus hijos, los liberan del control, evitan el uso de las restricciones y el castigo (Torío, Peña e Inda, 2008), ceden en su autoridad y les traspasan la 
responsabilidad de decidir a sus hijos (Valdivia, 2010). El hijo/a pasa a ser dueño de la casa, de las decisiones y a veces también de las decisiones de sus padres. Según refieren en su estudio Cerezo, Casanova, de la Torre y Carpio (2011), los alumnos con padres considerados tanto permisivos como democráticos utilizan en mayor medida estrategias de aprendizaje autorregulado, incidiendo esto positivamente en el desempeño académico de los alumnos. En estudios recientes realizados en España el estilo parental considerado más idóneo resultó ser el permisivo (Fuentes, García, Gracia \& Alarcón, 2015; García \& Gracia, 2010; Pérez Alonso-Geta, 2012). Asimismo en Portugal, un estudio refiere que los adolescentes de familias indulgentes puntuaron igual o mejor que los de familias democráticas en ajuste psicosocial (Rodrigues, Veiga, Fuentes \& García, 2013).

\section{Estilo autoritario}

Los padres con estilo autoritario valoran la obediencia (Martínez et al., 2013), como una virtud, mantienen a sus hijos subordinados y restringen su autonomía, provocando problemas en su adaptación social y una disminución de la seguridad en sí mismos (Torío, Peña \&Inda, 2008).

En cuanto a los hijos sus problemas se plantean a nivel emocional, debido al escaso apoyo recibido, los hijos se muestran tímidos, tienen mínima expresión de afecto con sus pares, pobre interiorización de valores, son irritables, vulnerables a las tensiones y poco alegres (Maccoby y Martin, 1983). Este estilo es un factor de riesgo para el padecimiento en los hijos de síntomas depresivos (Andrade, Bentancourt y Vallejo, 2012; Ato, Galián \& Huéscar, 2007; Richaud de Minzi, 2005; Vallejo, Osorno \& Mazadiego, 2008) y para el consumo de sustancias (Martínez et al., 2013). Asimismo se ha relacionado el estilo autoritario y el negligente con el estado nutricional de los adolescentes, asociándolo con malnutrición por exceso o déficit (Alzate \& Cánovas, 2013).

En un estudio llevado adelante por De la Torre, García-Linares y Casanova (2014), refieren que la percepción por parte de los adolescentes de un estilo parental autoritario se encuentra asociada a mayores 
manifestaciones de agresividad. En ese mismo sentido García-Linares (2014), refiere en su estudio que entre las variables que mejor predicen la agresividad se encuentran las prácticas educativas rígidas e indulgentes. Por su parte Patterson $(1982,2002)$, manifiesta que las prácticas de crianza coercitivas son un factor determinante en el comportamiento antisocial del niño. En ese mismo sentido y a partir del estudio realizado por Patterson (1982, 2000), Prinzie et al., (2004), concluyen en su estudio que las prácticas parentales con características coercitivas y un disciplinamiento imprudente se relacionan con problemas externalizantes en sus hijos (agresividad, hiperactividad o delincuencia). En ese mismo sentido Iglesias y Romero (2009), refieren una relación entre este estilo y las alteraciones externalizantes.

Es frecuente que cuando el control es muy severo los hijos terminen rebelándose ante sus padres, especialmente al principio de la adolescencia, momento en el que suelen buscar una mayor libertad y autonomía, mientras que sus padres y madres intentan seguir manteniendo un control estricto (Segura \& Mesa, 2011).

Más allá de estas clasificaciones de estilos parentales, que son referencia en numerosos estudios, hoy contamos con nuevos enfoques para comprender las relaciones familiares como por ejemplo: el modelo de construcción conjunta o influencias múltiples, desarrollado por Palacios (1999). En este modelo cobra vital importancia la bidireccionalidad de la relación entre padres e hijos y además se considera importante que las prácticas educativas se adecuen a la edad de los hijos y promuevan su desarrollo (Ceballos \& Rodrigo, 1998).

Para recoger el concepto de bidireccionalidad, por su parte, Oliva et al. (2008), deciden utilizar el nombre de estilos relacionales, en lugar de estilos parentales.

\section{Objetivo}

El objetivo principal consiste en identificar los estilos parentales, abarcando tanto la percepción de los padres sobre las prácticas educativas concretas, como la percepción que sobre las mismas, tienen sus hijos siguiendo el concepto de bidireccionalidad. De acuerdo a la 
literatura consultada, se espera: 1 . Encontrar discrepancias entre ambas percepciones 2. Identificar algunas diferencias en los estilos parentales, según variables sociodemográficas tales como nivel educativo y composición familiar. 3. Identificar diferencias en la percepción de los hijos sobre los estilos educativos, de padres y madres.

\section{Método}

\section{Participantes}

La muestra está constituida por 85 familias, en las cuales aparecen 107 referentes (padres y madres) y 85 preadolescentes. Además hay 22 adolescentes que contestan solo ellos los cuestionarios, sin sus referentes haciendo un total de 107 adolescentes. Estos adolescentes cursaron primer año de educación secundaria durante el año lectivo 2014 en cuatro colegios católicos de la ciudad de Montevideo. El total de familias que cumplían con la condición fue de 156, por lo que se tuvo una respuesta de casi el 55\%.

De los 107 padres y madres integrantes de la muestra, 63 cumplimentaron el instrumento de forma individual, como único adulto de la familia responsable del llenado de los cuestionarios. Ya sea por ser el único adulto en la familia o por una decisión familiar. Los otros 44 adultos (22 familias restantes) completaron los cuestionarios en forma conjunta (padre y madre) y aparecen ambos como responsables de su llenado.

El cuestionario de información sociodemográfica se les aplicó a todos los adultos que participaron del estudio.

A nivel descriptivo los 107 adultos alcanzan una edad media de 42.60 y una DT de 6.21, con una edad mínima de 30 años y una edad máxima de 66 años. En cuanto a las 78 madres (72,9\%), las mismas alcanzan una edad media de 41.53 , una DT de 5.89 y una edad mínima de 30 y una edad máxima de 54 años. Por su parte los 29 padres $(27,1 \%)$, tienen una edad media de 45.48 con una DT de 6.22, una edad mínima de 33 y una edad máxima de 66 años. Cincuenta y nueve de las madres del estudio, trabajan entre 6 y 10 horas diarias, 
mientras que 10 tienen una carga horaria laboral aún mayor. Seis progenitores alcanzaron nivel primaria $(5,60 \%)$, cuatro $(3,7 \%)$ no completaron ciclo básico, $53(49,5 \%)$ se ubican entre ciclo básico completo y preuniversitario, los 44 restantes (41\%) alcanzaron la universidad que solo $23(21,50 \%)$ finalizaron.

En cuanto a la configuración familiar reportada, 60 adultos $(56 \%)$ viven en familia nuclear, $22(20,5 \%)$ en familia monoparental, 9 $(8.4 \%)$ en familia ensamblada y $15(14,01 \%)$ en familia extensa.

De los datos sociodemográficos que surgen del estudio el $70 \%$ de las configuraciones familiares responden a modelos tradicionales familiares (56\% familias nucleares y $14 \%$ a familias extensas). Tengamos en cuenta que en América Latina hay una disminución de las formas tradicionales de convivencia (familia nuclear y familias extensas) (Arriagada, 2005: 2006; Organización Internacional del Trabajo/Programa de Naciones Unidas para el Desarrollo, 2009).

Asimismo cabe consignar que en Uruguay se percibe un incremento de los hogares monoparentales, producto básicamente del aumento de los divorcios, pasando de una tasa de 18,7\% para el ańo 1985 a una tasa de 33,7\% en el ańo 2002 (Cabella, 2007). En particular en Uruguay, las configuraciones monoparentales parecen encontrarse emparentadas con la capacidad de algunas mujeres de solventar los costos económicos que conlleva el mantenimiento de un hogar (Cabella, 2007). Encontramos tres elementos que colaboran en la reconfiguración familiar en nuestro país y estos son, el aumento de los divorcios, el envejecimiento de la población y la disminución de la fecundidad (Cabella, 2006; Paredes, 2003).

En cuanto a las horas de trabajo de las madres del estudio, tengamos presente que las mujeres mantienen un promedio de horas de trabajo remunerado similar al trabajo remunerado de los hombres/ padres del estudio. Es de hacer notar que según el Instituto Nacional de Estadística (2013), las mujeres prácticamente duplican a los hombres en horas de trabajo no remunerado.

Es por ello que llaman la atención las configuraciones familiares tradicionales encontradas, quizás vinculadas a la pertenencia de estas 
familias a colegios católicos de Montevideo, punto que seria interesante profundizar en futuras investigaciones.

En tanto los 107 hijos e hijas, vemos que alcanzan una edad media de 12.10 y una DT de 0.33 . De estos 107 sujetos, el 43\% son mujeres con un promedio de edad de 12.13 y una DT de 0.4 . En tanto los varones son el $57 \%$ con un promedio de edad de 12.08 y una DT de 0.27.

La elección realizada para esta investigación de alumnos de primer año de educación secundaria o sea adolescentes de 12 y 13 años y sus padres, tiene relación con la etapa de pasaje de la educación primaria a la educación secundaria. Se trata de un cambio trascendente para los chicos y sus padres, en la forma de relacionarse que establecen estos con sus hijos y con las instituciones educativas a las que estos concurren. Los adolescentes suelen preguntarse hacia dónde van, por los valores, las normas, sus ideales, sobre su proyecto de vida, traslada esa interrogante a los integrantes de su familia en particular a sus padres (Vidal, 2001). Por lo general, los adolescentes solicitan que se tengan nuevas consideraciones, más acordes a su nuevo estatus.

\section{Medidas e instrumentos de medición}

Cuestionario de Información Sociodemográfica: Se solicita a los participantes datos relativos a la conformación de la familia (constitución, estado civil, rol y edad de todos los miembros de la familia), e información sobre dos variables asociadas al contexto socioeducativo de las mismas (nivel educativo y ocupación de los padres y madres). Estos datos se estudiarán con la pretensión de lograr una caracterización básica de las familias que permita profundizar en el análisis de los estilos parentales.

Escala de Afecto y Escala de Normas y Exigencias (Bersabé, Fuentes \& Motrico, 2001): En estas escalas se toman en consideración las sugerencias realizadas por (Palacios, 1999; Ceballos \& Rodrigo, 1998). Se tiene en cuenta entonces tanto la perspectiva de los padres, como la de los hijos y además se utilizan las escalas en una etapa específica del ciclo vital, la adolescencia. Estas escalas han sido utilizadas en variadas investigaciones, que ponen en relación los estilos parentales con 
agresividad en adolescentes (De la Torre et al., 2014); inteligencia emocional, (Rucalcaba, Gallegos, Robles, Morales \& González, 2012), desempeño académico (Tilano, Henao \& Restrepo, 2009).

La Escala de Afecto (EA) está compuesta por 20 items divididos en dos factores:

- Afecto-comunicación. Afecto, interés y comunicación que manifiestan los padres a sus hijos/as. Ejemplo: $<<$ Soy cariñosola con mi hijola>>.

- Crítica-rechazo. Crítica, rechazo y falta de confianza de los padres hacia sus hijos/as. Ejemplo: <<Siento que es un estorbo para mi>>.

Cada uno de los factores consta de 10 ítems que se contestan en una escala tipo Likert con 5 grados de frecuencia (nunca, pocas veces, algunas veces, a menudo y siempre). La puntuación total de cada factor está comprendida entre 10 y 50.

La Escala de Normas y Exigencias (ENE) está formada por 28 items divididos en tres factores, referentes a la forma que tienen los padres de poner las normas a los hijos/as y exigir su cumplimiento:

- Forma nductiva. Los padres explican a sus hijos/as el establecimiento de las normas y les exigen su cumplimiento teniendo en cuenta las necesidades y posibilidades de estos. Ejemplo: $<<$ Tengo en cuenta las circunstancias ante de castigarle >>.

- Forma rígida. Los padres imponen a sus hijos/as el cumplimiento de las normas y mantienen un nivel de exigencias demasiado alto o inadecuado a las necesidades de estos. Ejemplo: <<Intento controlar su vida en todo momento >>.

- Forma indulgente. Los padres no ponen normas ni limites a la conducta de sus hijos/as y si lo hacen no exigen su cumplimiento. Ejemplos: $<<$ Si me desobedece, no pasa nada $>>$.

Los dos primeros factores cuentan con 10 items y el tercero se encuentra compuesto por 8. La puntuación total de los dos primeros factores se encuentra comprendida entre 10 y 50; la del tercer factor se 
encuentra comprendida entre 8 y 40. En la versión que contestan los hijos/as, en cada uno de los 5 factores se aportan dos puntuaciones: una referida al padre y otra a la madre. En la versión de los hijos, se evalúa la percepción que el adolescente tiene del estilo educativo de su padre y de su madre. En la versión de los padres, estos contestan los ítems refiriéndose a cómo es su conducta concreta con su hijo/a. Las propiedades psicométricas de las escalas (EA y ENE) se presentan en Bersabé et al., (2001). La consistencia interna (alpha de Cronbach) de cada uno de los factores varió entre 0,60 y 0,90 .

\section{Procedimiento}

Se llevó a cabo una investigación de carácter cuantitativa: descriptivo y comparativo (Hernández Sampieri, Fernández-Collado \& Baptista Lucio, 2006). Para el presente estudio, se trabajó con dos generaciones: padres e hijos. Para acceder a la muestra se tomó contacto a través de una entrevista personal con la dirección de cada centro educativo y se envío una carta dirigida a los padres de los alumnos en la que se explicaban brevemente los objetivos del estudio y se solicitaba su aprobación para llevar a cabo la aplicación de las pruebas. El procedimiento utilizado incorporaba la autorización expresa de los padres para la participación de sus hijos. La aplicación de los instrumentos se llevó a cabo de forma colectiva en los mismos centros educativos, en el espacio del aula y en el horario habitual de clase, en el caso de los adolescentes. Las instrucciones sobre los cuestionarios se explicaron oralmente, a la vez que se fueron leyendo los ítems para facilitar la comprensión. Se solicitó también consentimiento para la participación a los adolescentes. En el caso de los adultos referentes, se les enviaron los cuestionarios a los efectos de ser completados y devueltos a la institución educativa, junto con los consentimientos informados. El presente estudio forma parte de una línea de investigación mayor, que cuenta con la aprobación del Comité de Ética de la Facultad de Psicología de la Universidad Católica del Uruguay. 


\section{Análisis de datos}

El análisis de los datos se realizó con el programa estadístico SPSS versión 17.0. Para la realización de los análisis se tuvo en cuenta las siguientes variables predictoras, tanto la perspectiva de los preadolescentes como la de las madres y padres, en los factores: afecto, crítica, inductiva, rígida e indulgente. Los datos obtenidos fueron sometidos a un análisis estadístico descriptivo, comparativo y correlacional, mediante las pruebas t de Student, U de Mann-Whitney, ANOVA, Welch y Brown-Forsythe, Games-Howell, previo análisis de normalidad mediante Kolmogorov-Smirnov y Homocedasticidad (prueba de igualdad de varianzas de Levene).

\section{Resultados}

Previo a poner a prueba las hipótesis que se desprenden de los objetivos específicos, se analizó la normalidad de la variable estilos parentales divididos en cinco factores, para padres e hijos. Los valores del estadístico Kolmogorov-Smirnov para padres, en los cinco factores estudiados (afecto, crítica, inductiva, rígida e indulgente) dieron niveles de significación de $p=.102, p=.069, p=.091, p=.398, p=.304$ ( $>$ a .05) para cada factor respectivamente, lo cual indica que debe aceptarse la hipótesis de normalidad para la muestra de padres.

$\mathrm{Al}$ poner a prueba las escalas de los hijos y su percepción sobre padres y madres se obtuvieron niveles críticos menores a .05 en las escalas de afecto sobre padres $(p=.023)$, en la de crítica sobre padres $(p=.001)$ y en la escala de afecto sobre madres $(p=.009)$, lo cual indica que debe rechazarse la hipótesis de normalidad concluyendo que los datos no se distribuyen normalmente. Por lo tanto, se utilizan estadísticos paramétricos para padres y no paramétricos para hijos.

En primer lugar, para saber si existen diferencias significativas entre el nivel educativo formal alcanzado por los padres y la elección del estilo educativo parental, se procede a utilizar el estadístico ANOVA. Dado que el estadístico ANOVA de un factor se basa en el cumplimiento de 
los supuestos de normalidad y homocedasticidad, se realiza la prueba de Levene de igualdad de varianzas para cada uno de los factores, obteniendo únicamente niveles de significación menores a .05 en las escalas crítica $(p=.017)$ e inductiva $(p=.042)$, por lo cual se utilizan los estadísticos robustos de Welch y Brown-Forsythe para dichas escalas.

Para la escala crítica, dado que el nivel primaria de la variable Nivel Educativo alcanzado presenta un solo caso, no fue posible realizar el análisis. En tanto, para la escala Inductiva, los estadísticos robustos presentan un nivel crítico de $p=.009$ y $p=.490$ en Welch y Brown-Forsythe respectivamente. Se realiza el análisis post hoc mediante el estadístico Games-Howell, para determinar entre qué grupos radican las diferencias, obteniendo diferencias significativas en la escala Inductiva entre quienes alcanzaron el nivel primaria $(M=47.5)$ y el nivel terciario universitario $(M=44.24)$, con un nivel crítico asociado $p=.027$. Dado que se cumple el supuesto de homocedasticidad en las escalas de Afecto $(p=.347)$, Rígida $(p=.304)$ e Indulgente $(p=.149)$, utilizamos ANOVA de un Factor para dichas escalas. Los resultados del ANOVA fueron $p=.336$ para Afecto, $p=.043$ para Rígida y $p=.388$ para Indulgente.

Al realizar el análisis post hoc mediante la prueba de Tukey de la escala Rígida se obtiene un nivel de significación de $p=.431$, concluyendo que las diferencias en la variable Nivel Educativo alcanzado no son significativas para dicha escala.

Para identificar si existen diferencias significativas entre la composición familiar y la elección del estilo educativo parental, se procede a utilizar el estadístico ANOVA, previo análisis de homocedasticidad.

De la prueba de igualdad de varianzas de Levene, se obtienen niveles de significación menores a .05 , en las escalas de afecto $(p=.001)$ e inductiva $(p=.030)$, por lo que se utilizan los estadísticos robustos, y cumpliéndose el supuesto para las escalas Crítica $(p=.481)$, Rígida $(p=.326)$ e Indulgente $(p=.280)$ por lo que se utiliza ANOVA de un F. Del análisis efectuado mediante los estadísticos robustos Welch y Brown-Forsythe se obtiene para la escala de Afecto, niveles de significación de $p=.171$ y $p=.397$ para cada estadístico respectivamente. Para la escala Inductiva, se obtiene niveles de significación de $p=.178$ 
y $p=.365$ respectivamente, por lo tanto, se concluye que no existen diferencias significativas en los promedios de las escalas de Afecto e Inductiva según la composición familiar. Para las escalas Crítica, Rígida e Indulgente se obtienen mediante el análisis ANOVA de un F, valores de significación mayores a $.05,(p=.449, p=.066$ y $p=.145$ respectivamente), por lo cual no existen diferencias significativas en ninguna de las escalas estudiadas según la composición familiar.

Para analizar si los padres y las madres tienen una percepción diferente en cada uno de los factores de la variable Estilos Parentales, se utilizó la prueba t de Student, como se presentan los datos descriptivos y la comparación de medias en la Tabla 1.

\section{Tabla 1}

Diferencias entre padres y madres en los factores de la variable Estilos Parentales

\begin{tabular}{|c|c|c|c|c|c|c|c|}
\hline \multirow[b]{2}{*}{ Escalas } & & \multicolumn{2}{|c|}{ Padres } & \multicolumn{2}{|c|}{ Madres } & \multirow[t]{2}{*}{ Levene } & \multirow[t]{2}{*}{$t$} \\
\hline & & $M$ & $D E$ & $M$ & $D E$ & & \\
\hline \multirow{2}{*}{ EA } & Afecto & 46.60 & 2.793 & 45.82 & 3.989 & .520 & -.423 \\
\hline & Crítica & 13.50 & 1.049 & 15.45 & 3.241 & $5.252^{*}$ & $3.091^{*}$ \\
\hline \multirow{3}{*}{ ENE } & Inductiva & 43.57 & 4.198 & 44.30 & 4.320 & .062 & .419 \\
\hline & Rígida & 29.71 & 5.908 & 25.83 & 6.968 & .758 & -1.404 \\
\hline & Indulgente & 14.43 & 4.650 & 14.02 & 4.028 & .064 & -.247 \\
\hline
\end{tabular}

${ }^{*} p<0.05$

Se puede observar en la Tabla 1, que la prueba $t$ para muestras independientes confirma diferencias estadísticamente significativas en la percepción de los padres y las madres, correspondiente al factor crítica/rechazo, con un nivel de significancia de 0.006 .

En cuanto a la percepción de padres y madres, según sean sus hijos varones o mujeres, se encuentran diferencias estadísticamente significativas en la percepción de los padres según sean los hijos de sexo masculino o femenino, únicamente en la subescala Inductiva, con un $p=0.006$, como se observa en la Tabla 2 . 
Estilos relacionales parentales: Estudio con adolescentes y sus padres / Capano et al.

\section{Tabla 2}

Diferencias en la percepción de los padres según el sexo de sus hijos

\begin{tabular}{lcccrrrr}
\hline & \multicolumn{2}{c}{ Hijas } & \multicolumn{2}{c}{ Hijos } & \multirow{2}{*}{ Levene } & Prueba $t$ \\
\cline { 2 - 5 } Escalas & $M$ & $D E$ & $M$ & $D E$ & & \\
\hline Afecto & 47.00 & 2.492 & 45.28 & 4.386 & 2.823 & 1.613 \\
Crítica & 15.32 & 3.329 & 15.18 & 3.046 & .111 & .157 \\
Inductiva & 46.14 & 3.357 & 43.00 & 4.386 & 1.604 & $2.865^{*}$ \\
Rígida & 26.20 & 7.575 & 26.37 & 6.589 & & .747 & -.093 \\
Indulgente & 14.45 & 4.009 & 13.83 & 4.141 & & .004 & .562 \\
\hline
\end{tabular}

${ }^{*} p<0.05$

Al comparar la percepción de hijos e hijas con respecto a la percepción de padres y madres, se encuentra diferencias entre la percepción de los hijos e hijas con respecto a la de los padres, para las escalas de Afecto e Indulgente. También se observan diferencias significativas en la percepción de hijos e hijas respecto a las madres en las escalas de Afecto, Inductiva e Indulgente, como se observa en la Tabla 3.

\section{Tabla 3}

Diferencias en la percepción de hijos e hijas respecto a la percepción de padres y madres según los factores de la variable Estilos Parentales

\begin{tabular}{|c|c|c|c|c|c|c|c|c|c|}
\hline \multirow[t]{2}{*}{$\begin{array}{l}\text { Estilo } \\
\text { parental }\end{array}$} & & \multicolumn{2}{|c|}{$\begin{array}{l}\text { Versión hijas } \\
\text { e hijos } \\
\end{array}$} & \multicolumn{2}{|c|}{$\begin{array}{c}\text { Versión padres y } \\
\text { madres }\end{array}$} & \multirow[t]{2}{*}{$U$} & \multirow[t]{2}{*}{$W$} & \multirow[t]{2}{*}{$Z$} & \multirow[t]{2}{*}{$p$} \\
\hline & & $M$ & $D E$ & $M$ & $D E$ & & & & \\
\hline \multirow{5}{*}{ Paterno } & Afecto & 38.18 & 9.79 & 46.60 & 2.79 & 82.5 & 3910.5 & -2.33 & $0.02^{*}$ \\
\hline & Crítica & 15.83 & 5.73 & 13.50 & 1.05 & 210.0 & 231.0 & -0.84 & 0.40 \\
\hline & Inductiva & 40.14 & 8.36 & 43.57 & 4.20 & 247.0 & 4163.0 & $-0,87$ & 0.38 \\
\hline & Rígida & 26.16 & 6.57 & 29.71 & 5.91 & 214.0 & 4042.0 & -1.31 & 0.19 \\
\hline & Indulgente & 17.68 & 4.12 & 14.43 & 4.65 & 147.0 & 175.0 & -2.17 & $0.03^{*}$ \\
\hline \multirow{5}{*}{ Materno } & Afecto & 42.68 & 7.219 & 45.82 & 3.989 & 1016.5 & 4844.5 & 5.31 & $0.00^{*}$ \\
\hline & Crítica & 26.10 & 3.471 & 15.45 & 3.241 & 1983.5 & 5899.5 & -0.78 & 0.44 \\
\hline & Inductiva & 42.03 & 6.332 & 244.30 & 4.320 & 1608.0 & 5524.0 & -2.63 & $0.01^{*}$ \\
\hline & Rígida & 27.00 & 6.361 & 25.83 & 6.968 & 2091.0 & 3366.0 & -0.38 & 0.71 \\
\hline & Indulgente & 17.63 & 4.090 & 14.02 & 4.028 & 1085.5 & 2360.5 & -4.61 & $0.00^{*}$ \\
\hline
\end{tabular}


Para analizar estas comparaciones se utilizó la prueba U de MannWhitney acompañada de la corrección de Bonferroni para controlar la tasa de error tipo 1 (rechazo de hipótesis nula que es verdadera). Esta corrección implica dividir el nivel de significación .05 entre el número de comparaciones a realizar, en este caso, dos (Pardo \& Ruiz, 2002). El nivel crítico corregido por Bonferroni es para las escalas de Afecto e Inductiva en Padres versus hijos/as $.06 / 2=.03$ y $.05 / 2=.025$ respectivamente, como se observa en la Tabla 4.

\section{Tabla 4}

Diferencias en la percepción de hijos versus hijas según los estilos parentales de sus padres y madres respectivamente

\begin{tabular}{|c|c|c|c|c|c|c|c|c|c|}
\hline & & \multicolumn{2}{|l|}{ Hijas } & \multicolumn{2}{|l|}{ Hijos } & \multirow[t]{2}{*}{$U$} & \multirow[t]{2}{*}{$W$} & \multirow[t]{2}{*}{$Z$} & \\
\hline & & $M$ & $D E$ & $M$ & $D E$ & & & & \\
\hline \multirow{5}{*}{$\begin{array}{l}\text { Sobre } \\
\text { padres }\end{array}$} & Afecto & 35.36 & 11.46 & 40.18 & 7.95 & 699.5 & 1365.5 & -1.89 & $0.06^{*}$ \\
\hline & Crítica & 16.11 & 6.08 & 15.64 & 5.51 & 931.0 & 1634.0 & -0.11 & 0.92 \\
\hline & Inductiva & 39.43 & 8.62 & 40.67 & 8.22 & 864.0 & 1567.0 & -0.67 & $0.05^{*}$ \\
\hline & Rígida & 25.49 & 6.05 & 26.64 & 6.92 & 806.0 & 1472.0 & -0.97 & 0.33 \\
\hline & Indulgente & 17.79 & 4.26 & 17.78 & 4.06 & 825.0 & 1420.0 & -0.07 & 0.94 \\
\hline \multirow{5}{*}{$\begin{array}{l}\text { Sobre } \\
\text { madres }\end{array}$} & Afecto & 42.46 & 7.84 & 42.82 & 6.87 & 958.0 & 1588.5 & -0.33 & 0.97 \\
\hline & Crítica & 26.31 & 3.81 & 25.97 & 3.26 & 938.5 & 2478.5 & -0.42 & 0.68 \\
\hline & Inductiva & 41.21 & 6.70 & 42.67 & 6.01 & 937.5 & 1798.5 & -1.14 & 0.26 \\
\hline & Rígida & 26.94 & 6.26 & 27.04 & 6.49 & 1118.0 & 2714.0 & -0.02 & 0.99 \\
\hline & Indulgente & 17.55 & 3.92 & 17.68 & 4.24 & 933.5 & 1636.5 & -0.24 & 0.81 \\
\hline
\end{tabular}

${ }^{*} p<0.05$

Según los resultados, encontramos diferencias significativas en la forma en que perciben las hijas y los hijos a sus padres en los factores Afecto e Inductiva. No se encuentran diferencias significativas al comparar la percepción de las madres con la de los hijos o hijas. 
Estilos relacionales parentales: Estudio con adolescentes y sus padres / Capano et al.

\section{Discusión}

Los resultados del presente trabajo son consistentes con otros estudios realizados referidos a que la percepción de padres e hijos sobre los estilos parentales no coincide totalmente (Alonso \& Román, 2005; Bersabé et al., 2001; Fuentes et al., 2003; González \& Landero, 2012; Rodriguez, Pérez-Fuentes y Gázquez, 2013; Tilano et al. 2009) percibiéndose los padres más democráticos de lo que los consideran sus hijos. Justamente, las diferencias en cuanto a la percepción de padres e hijos llevó a Bersabé et al. (2001), a plantear la necesidad de evaluar los estilos parentales desde ambas perspectivas. En este sentido, Smetana (1995), refirió que los adolescentes percibían a sus padres como permisivos y autoritarios, mientras los padres se percibían a sí mismos como democráticos. Las diferencias generales entre padres/madres e hijos/ hijas relacionados a la escala inductiva, se presenta en el contraste entre la percepción de hijos e hijas y sus madres, percibiéndose estas más inductivas que la percepción que tienen sus hijos e hijas de ellas. En este sentido, cabe consignar que los hijos/hijas perciben a sus padres menos cariñosos y más indulgentes, en tanto a las madres las perciben menos cariñosas, menos inductivas y más indulgentes.

La comparación realizada entre padre y madre en relación con los cinco factores estudiados, deja en evidencia una diferencia significativa en el factor crítica y rechazo. Siendo la madre quien alcanza los niveles más altos a diferencia del estudio realizado por Tilano et al., (2009) donde el mayor nivel de crítica/rechazo recae sobre el padre. Otros estudios muestran como las madres en relación a los padres se presentan con una mayor influencia, aceptación incondicional y más implicadas con sus hijos e hijas (Etxebarria, Apodaca, Fuentes, López \& Ortiz, 2009; Laible \& Carlo, 2004). Esta influencia se da tanto respecto a un estilo de disciplina inductiva como al estilo de crianza más punitivo (Tur-Porcar, Mestre, Samper \& Malonda, 2012). Las diferencias significativas en relación con la percepción que hijos e hijas tienen de forma conjunta o individualmente sobre las madres quizás tenga relación con el hecho que las mujeres tienen mayor exposición e implicación en la crianza diaria 
de sus hijos/hijas, específicamente en los temas relacionados a la vida cotidiana (Parra \& Oliva, 2002) y al lugar relevante que presentan en la educación de sus hijos (Etxebarria et al., 2009), aún pese a que trabajan fuera del hogar. Asimismo cabe la posibilidad que esa mayor implicación de la madre en la crianza traiga aparejada situaciones conflictivas con sus hijos e hijas, siendo estas últimas quienes más discrepan con sus madres (Parra \& Oliva, 2002; Rodrigo, García, Máiquez \& Triana, 2005).

Asimismo se encontró que padres y madres se perciben significativamente más inductivos con sus hijas mujeres que con sus hijos varones. En referencia a este punto, Musitu y Cava (2001), refieren que respecto a los conflictos familiares, los varones tienden a la indiferencia y utilizan con frecuencia la mentira como estrategia, mientras que las mujeres si bien expresan en mayor medida su enojo, son más propensas a buscar el diálogo, especialmente con la madre. Dentro de una concepción bidireccional, este hecho puede convocar diferentes estrategias de los padres, según el género de los hijos.

De la comparación por sexo y en cuanto a la percepción que las hijas e hijos tienen sobre padre y madre, surge que las hijas perciben a sus padres significativamente menos carińosos e inductivos que los hijos. No hay diferencias significativas en la forma en que perciben hijos e hijas el estilo materno. Estos resultados difieren de otros estudios, donde las hijas perciben estilos parentales más adecuados que los hijos. Los hijos perciben a sus padres más rígidos, exigentes, con pautas educativas negativas, centradas en la irritabilidad, controladores con un mayor nivel de crítica y rechazo, mientras que las hijas manifiestan mayores puntuaciones en afecto, comunicación y disciplina inductiva (Fuentes et. al., 2001; Fuentes et al., 2003; García-Linares, Cerezo, de la Torre, Carpio \& Casanova, 2011; Mestre, Tur-Porcar, Samper, Nácher \& Cortés, 2007; Pastor, 2014; Polo del Río, Fajardo, Martín, Gómez \& León del Barco, 2012; Rodriguez, Del Barrio \& Carrasco, 2009; Tilano, et al., 2009).

Por su parte y con respecto al nivel educativo de los padres y su relación con el estilo parental, se encuentra una baja concordancia entre el presente estudio y otras investigaciones. El estudio arroja diferencias 
significativas en el factor inductivo, siendo los padres con educación primaria más inductivos que los padres con educación terciaria-universitaria. Cabe consignar que, según diversas investigaciones los padres con un nivel de educación más alto se manifiestan de forma más democrática que los padres con un bajo nivel educativo (Dornbusch, Ritter, Liedermann, Roberts \& Fraleigh, 1987), presentan mejores habilidades emocionales y una mayor comunicación afectiva (Ruvalcaba et al., 2012). Asimismo se ha manifestado que la educación de los padres se relaciona con altos niveles de responsividad, como los que se dan en los estilos autoritativo y permisivo (Glasgow, Dornbusch, Troyer \& Steinberg, 1997). Por su parte Casullo y Liporace (2008), refieren que los padres con primaria incompleta o completa presentan inconsistencias significativamente mayores que los padres con educación terciaria o universitaria. Sin embargo Etxebarria et al., (2009), refieren en su estudio una menor implicación de los padres y madres universitarios en cuanto al ejercicio de la parentalidad. Si bien los padres con enseñanza primaria fueron escasos en nuestro estudio, las diferencias halladas, y la existencia de otros estudios, dejan la puerta abierta a seguir investigando al respecto.

En cuanto a la configuración familiar, no se encontraron diferencias significativas en cuanto el estilo educativo. Este resultado puede ir en la dirección de estudios recientes, tales como el de Oliva y Arranz (2011) que no encontraron diferencias significativas en los estilos parentales en familias de cinco configuraciones diferentes.

Una de las limitaciones del presente estudio es que al ser respondidos los cuestionarios bajo la modalidad de autoadministración los participantes podrían haber respondido desde el deber ser y no desde cómo manifiestan realmente la conducta hacia sus hijos. Sería conveniente en ese sentido, incorporar otras técnicas tales como la observación directa del comportamiento de los padres hacia los hijos o las entrevistas con los padres y con los adolescentes, técnicas previstas para el estudio de estilos parentales (Arranz, Aspiroz et. al., 2004).

Consignamos como fortaleza el hecho que este es el primer estudio del que se tiene referencia en nuestro país sobre los estilos parentales 
en adolescentes desde esta perspectiva. Por lo tanto, se contribuye a abrir la discusión a partir del uso de un instrumento que cuenta con estudios a nivel internacional.

El estudiar los estilos parentales desde la perspectiva de padres e hijos, trae mayor riqueza a la discusión en las prácticas educativas diarias ya que los mayores desencuentros radican justamente en las diferentes visiones de autonomía y control que tienen ambas generaciones. El hecho de contar con instrumentos que capten la bidireccionalidad de las relaciones familiares y la percepción de padres e hijos sobre los estilos parentales, nos deja entonces en mejor situación para planificar intervenciones más ajustadas a la realidad que viven las familias actualmente. Siempre teniendo en cuenta que los estilos parentales, han demostrado ser una variable tomada en cuenta para estudiar el ajuste y bienestar psicológico de los hijos.

Debemos tener en cuenta que los participantes, hijos e hijas, recién están debutando en la adolescencia, por lo que en esta etapa, tanto ellos como sus padres están acomodándose a esta etapa vital, con expectativas de ambas partes y de parte de la sociedad, por ejemplo, referidas a las nuevas responsabilidades a asumir con el cambio del ciclo de enseñanza primaria al de secundaria. Se debe tener en cuenta, desde una perspectiva sistémica, que las relaciones entre padres e hijos son influenciadas y cambian, tanto por la madurez de los hijos como por la madurez de los padres (Araujo de Morais, Lima \& Fernandes, 2014).

Conocer la perspectiva sobre los estilos parentales de padres e hijos, puede dar datos interesantes justo en la adolescencia, etapa en la que van cobrando relevancia otros agentes de socialización tales como los amigos, los medios de comunicación y las redes sociales. Esto teniendo en cuenta que los diferentes agentes tendrán distintas visiones de los valores, fomentarán diversas habilidades e influirán en el autoconcepto de los adolescentes (Musitu y Cava, 2001). Es por ello que el estudio de los estilos relacionales parentales cobra mayor significado si se tienen en cuenta también los diferentes contextos en que tanto padres como hijos viven y se desenvuelven, ya que esos contextos pueden reforzar o contradecir dichos estilos. 
Estilos relacionales parentales: Estudio con adolescentes y sus padres / Capano et al.

\section{Referencias}

Alonso, J. \& Román, J. M. (2005). Prácticas educativas familiares y autoestima. Psicothema, 17(1), 76-82.

Alzate, T. \& Cánovas, P. (2013). Estado nutricional infantil y estilos educativos familiares: apreciación de expertos. Perspectivas en Nutrición Humana, 12(2), 185-199.

Andrade, P., Betancourt, D. \& Vallejo, A. (2012). Prácticas parentales y sintomatología depresiva en adolescentes. Salud Mental, 35, 29-36.

Araújo de Morais, N., Lima, R. \& Fernandes, J. (2014). Adolescencia e contexto familiar. En L.F. Habigzang, E. Diniz y S.Koller (Orgs.), Trabalhando com adolescentes. Teoria e intervenção psicológica (pp. 101-117). Porto Alegre: Artmed.

Arranz, E., Aspiroz, A., Arantzazu, B., Malla, R., Manzano, A., Martí, J. L., Olabarrieta, F., Oliva, A. \& Parra, A. (2004). Contexto familiar facilitador del desarrollo psicológico. Descripción, evaluación e intervención. Familia y desarrollo psicológico (pp. 170-219). Madrid: Pearson Educación.

Arranz, E., Bellido, A., Manzano, A., Martín, J. L. \& Olabarrieta, F. (2004). Contexto familiar y desarrollo psicológico durante la infancia. En E. Arranz (Coord.), Familia y desarrollo psicológico. (pp. 70-95). Madrid: Pearson Educación.

Arriagada, I. (Ed.) (2005). Politicas hacia las familias, protección e inclusión sociales. Serie Seminarios y Conferencias, 46. Santiago de Chile: CEPAL/UNFPA.

Arriagada, I. (2006). Trasformaciones de las familias en América Latina. En C. Fassler (Coord.), Familias en cambio en un mundo en cambio (pp. 63-79). Uruguay: Trilce.

Ato, E., Galián, Ma. D. \& Huéscar, E. (2007). Relaciones entre estilos educativos, temperamento y ajuste social en la infancia: una revisión. Anales de Psicología, 23(1), 33-40.

Baumrind, D. (1966). Effects of Authoritative Parental Control on Child Behavior. Child Development, 37(4), 887-907. http:// dx.doi.org/10.2307/1126611 
Becoña, E., Martínez, U., Calafat, J., Fernández-Hermida, J. \& Secades-Villa, R. (2012). Parental styles and drug use: A review. Drugs Educ Prev Pol, 19(1), 1-10. http://dx.doi.org/10.3109/09 687637.2011.631060

Bersabé, R. M., Fuentes, M. J. \& Motrico, E. (2001). Análisis psicométricos de dos escalas para evaluar estilos parentales. Psicothema 13(4), 678-684.

Bertalanffy, L. V. (1968). Systems theory: foundations, developments, applications. Nex York: Braziller.

Bornstein, M. H. \& Zlotnik, D. (2008). Parenting styles and their effects. En M. M. Haith y J. B. Benson (Eds.), Encyclopedia of infant and early childhood development (vol. 2, 496-509). Oxford: Academic Press. http://dx.doi.org/10.1016/b978-0123708779.00118-3

Cabella, W. (2006). Los cambios recientes de la familia uruguaya: la convergencia hacia la segunda transición demográfica. En C. Fassler (Coord.), Familias en cambio en un mundo en cambio (pp. 80-108). Uruguay: Trilce.

Cabella, W. (2007). El cambio familiar en Uruguay: una breve reseña de las tendencias recientes. Montevideo: UNFPA.

Cano, A. J., Solanas, S. E., Marí-Klose, M. \& Marí-Klose, P. (2012). Factores de riesgo psicosociales en el consumo de tabaco de los adolescentes: Estados de ánimo negativos, grupo de iguales y estilos parentales. Adicciones, 24(4), 309-318. http://dx.doi. org/10.20882/adicciones.81

Carpio, M. de la V., García Linares, M., de la Torre, M., Cerezo, M. \& Casanova, P. (2014). Consistencia e inconsistencia en los estilos de padres y madres y ajuste psicológico en los adolescentes. International Journal of Developmental and Educational Psychology, 2(1), 447-456.

Casullo, M. M. \& Liporace, M. F. (2008). Percepción sobre estilos e inconsistencia parentales en adolescentes argentinos. Estudos de Psicologia, 25(1), 3-9. http://dx.doi.org/10.1590/ s0103-166x2008000100001 
Estilos relacionales parentales: Estudio con adolescentes y sus padres / Capano et al.

Ceballos, E. \& Rodrigo, M. J. (1998). Las metas y estrategias de socialización entre padres e hijos. En M. J. Rodrigo y J. Palacios (Comps.), Familia y desarrollo humano (pp. 225-243). Madrid: Alianza.

Ceberio, M. R. (2013). El cielo puede esperar. Argentina: Morata.

Cerezo, M. T., Casanova, P., de la Torre, M. \& Carpio, M. de la V. (2011). Estilos educativos paternos y estrategias de aprendizaje en alumnos de Educación Secundaria. European Journal of Education and Psychology, 4(1), 51-61.

Climent, G. (2009). Voces, silencios y gritos: Los significados del embarazo en la adolescencia y los estilos parentales educativos. Revista Argentina de Sociología, 12/13, 186-213.

Cortés, M. R., Cantón, J. \& Cantón Cortés, D. (2011). Desarrollo socioafectivo en el contexto familiar. En J. Cantón Duarte, M. R. Cortés Arboleda y D. Cantón Cortés, Desarrollo socioafectivo y de la personalidad (pp. 213-259). Madrid: Alianza Editorial.

Darling, N. \& Steinberg, L. (1993). Parenting styles as context: an integrative model. Psychological Bulletin, 113, 487-496. http:// dx.doi.org/10.1037/0033-2909.113.3.487

De la Torre, M. J., García Linares, M. C. \& Casanova, P. F. (2014). Relaciones entre estilos parentales y agresividad en adolescentes. Electronic Journal of Research in Educational Psychology, 12(1), 147-170. http://dx.doi.org/10.14204/ejrep.32.13118

Dornbusch, S. M., Ritter, P. L., Liedermann, P. H., Roberts, D. F. \& Fraleigh, M. J. (1987). The relation of parenting style to adolescent school performance. Child Development, 58, 12441257. http://dx.doi.org/10.2307/1130618

Eisenberg, N., Zhou, Q., Spinrad, T. L., Valiente, C., Fabes, R. A. \& Liew, J. (2005). Relations Among Positive Parenting, Children's Effortful Control, and Externalizing Problems: A Three-Wave Longitudinal Study. Child Development, 76(5), 1055-1071. http://dx.doi.org/10.1111/j.1467-8624.2005.00897.x

Etxebarria, I., Apodaca, P., Fuentes, M. J., López, F. \& Ortiz, M. J. (2008). La crianza y la educación de los hijos en la sociedad 
actual: ¿lo estamos haciendo bien? Revista de Psicología Social, 2009, 24(1), 1-16.

Flaquer, L. (1998). El destino de la familia. Barcelona: Ariel.

Fuentes, M. C., Alarcón, A., García, F. \& Gracia, E. (2014). Consumo de alcohol, tabaco, cannabis y otras drogas en la adolescencia: efectos de la familia y peligros del barrio. Anales de Psicología. Recuperado de http://revistas.um.es/analesps/ article/viewFile/183491/173881. http://dx.doi.org/10.6018/ analesps.31.3.183491

Fuentes, M. C., García, F., Gracia, E. \& Alarcón, A. (2015). Los estilos parentales de socialización y el ajuste psicológico. Un estudio con adolescentes espańoles. Revista Psicodidáctica, 20(1), 117 138. http://dx.doi.org/10.1387/RevPsicodidact.10876

Fuentes, M. J., Motrico, E \& Bersabé, R. M. (2001). Diferencias entre padres y adolescentes en la percepción del estilo educativo parental: afecto y normas-exigencias. Apuntes de Psicología, 19(2), 235-250.

Fuentes, M. J., Motrico, E. \& Bersabé, R. M. (2003). Estrategias de socialización de los padres y conflictos entre padres e hijos en la adolescencia. Anuario de Psicología, 34(3), 385-400.

García, F. \& Gracia, E. (2010). ¿Qué estilo de socialización parental es el idóneo en España? Un estudio con niños y adolescentes de 10 a 14 años. Infancia y Aprendizaje, 33(3), 365-384. http://dx.doi. org/10.1174/021037010792215118

García Linares, M. C. (2014). Prácticas educativas paternas que predicen la agresividad evaluada por distintos informantes. Revista Latinoamericana de Psicología, 46(1-3), 198-210. http://dx.doi. org/10.1016/s0120-0534(14)70023-8

García Linares, M. C., Cerezo, M. T., de la Torre, M. J., Carpio, M. de la V. \& Casanova, P. F. (2011). Prácticas educativas paternas y problemas internalizantes y externalizantes en adolescentes españoles. Psicothema, 23(4), 654-659.

García Linares, M. C., de la Torre, M. J., Carpio, M. de la V., Cerezo, M. T. \& Casanova, P. F. (2014). Consistencia/inconsistencia en los estilos educativos de padres y madres, y estrés cotidiano en 
la adolescencia. Revista de Psicodidáctica, 19(2), 307-325. http:// dx.doi.org/10.1387/RevPsicodidact.7219

Gervilla, A. (2008). Familia y educación familiar: conceptos clave, situación actual y valores. Madrid: Narcea.

Gimeno, A. (2007). Las familias en la sociedad de la información y de la diversidad. En M. R. Buxarrais y M. P. Zeledón (Coords.), Las familias y la educación en valores democráticos. Retos y perspectivas actuales (pp. 99-126) Barcelona: Claret.

Glasgow, K. L., Dornbusch, S. M., Troyer, L. \& Steinberg, L. (1997). Parenting styles, adolescent's attributions, and educational outcomes in nine heterogeneous high school. Child Development, 68, 507-529. http://dx.doi.org/10.2307/1131675

González Tornaría, M. del L., Vandemeulebroecke, L. y Colpin, H. (2001). Pedagogía familiar. Aportes desde la teoría y la investigación. Montevideo: Trilce.

González, M. \& Landero, R. (2012). Diferencias en la percepción de estilos parentales entre jóvenes y adultos de las mismas familias. Summa Psicológica UST, 9(1), 53-64.

Gracia, E., Fuentes, M. C. y García, F. (2010). Barrios de riesgo, estilos de socialización parental y problemas de conducta en adolescentes. Intervención Psicosocial, 19(3), 265-278. http:// dx.doi.org/10.5093/in2010v19n3a7

Hernández, A. (1997). Familia, ciclo vital y psicoterapia sistémica breve. Bogotá: El Búho.

Hernández Sampieri, R., Fernández-Collado, C. \& Baptista Lucio, P. (2006). Metodología de la Investigación. México: McGraw Hill.

Iglesias, B. y Romero, E. (2009). Estilos parentales percibidos, psicopatología y personalidad en la adolescencia. Revista de Psicopatología y Psicologia Clínica, 14(2), 63-77. http://dx.doi.org/10.5944/ rppc.vol.14.num.2.2009.4067

Instituto Nacional de Estadística, (2013). Encuesta del uso del tiempo Recuperado de: http://www.ine.gub.uy/biblioteca/Uso\%20 del\%20Tiempo/USO\%20DEL\%20TIEMPO\%20Y\%20TRABAJO\%20NO\%20REMUNERADO.pdf 
Laible, D. J. \& Carlo, G. (2004). The differential relations of maternal and paternal support and control to adolescent social competente, self-worth, and sympathy. Journal of Adolescent Research, 19, 759-782. http://dx.doi.org/10.1177/0743558403260094

Lila, M. \& Gracia, E. (2005). Determinantes de la aceptación-rechazo parental. Psicothema, 17, 107-111.

Linares, J. L. (2009). La personalidad y sus trastornos desde una perspectiva sistémica. En Murueta, M. y Osorio, M. (Comp) Psicología de la Familia en países latinos del siglo XXI (pp. 101122). México: Amapsi.

López-Soler, C., Puerto, J., López-Pina, J. \& Prieto, M. (2009). Percepción de los estilos parentales e inadaptación en menores pediátricos. Anales de psicología, 25(1), 70-77.

Maccoby, E. E. \& Martín, J. A. (1983). Socialization in the context of the family: Parent-child interaction. En E.M Hetherington y P. H. (eds): Handbook of child psychology: Socialization, personality and social development, 4, 1-101. New York: Wiley.

Martínez, I., Fuentes, M., García, F. \& Madrid, I. (2013). El estilo de socialización familiar como factor de prevención o riesgo para el consumo de sustancias y otros problemas de conducta en los adolescentes españoles. Adicciones, 25(3), 235-242. http:// dx.doi.org/10.20882/adicciones. 51

Mestre, M. V., Tur-Porcar, A., Samper, P., Nácher, M. J. \& Cortés, M. T. (2007). Estilos de crianza en la adolescencia y su relación con el comportamiento prosocial. Revista Latinoamericana de Psicología Vol. 39 (2), 211-225.

Minuchin, S. (1974). Familias y terapia familiar. Barcelona: Gedisa.

Musitu, G. \& Cava, M. J. (2001). La familia y la educación. Barcelona: Octaedro.

Nardone, G., Giannotti, E. \& Rocchi, R. (2003). Modelos de familia. Conocer y resolver los problemas entre padres e hijos. Barcelona: Herder. 
Estilos relacionales parentales: Estudio con adolescentes y sus padres / Capano et al.

Oliva, A. \& Arranz, E. (Coords.) (2011). Nuevas familias y bienestar infantil. España: Secretariado de Publicaciones de la Universidad de Sevilla y Servicio editorial de la Universidad del País Vasco.

Oliva, A., Hidalgo, V., Martín, D., Parra, A., Ríos, M. y Vallejo, R. (2007). Programa de Apoyo a Madres y Padres de Adolescentes. Dpto. de Psicología Evolutiva y la Educación, Universidad de Sevilla, Andalucía Consejería de Salud. Recuperado de: http:// etfgranada.blogspot.com/2012/09/programa-de-apoyo-madresy-padres-de.html

Oliva, A., Parra, A. \& Arranz, E. (2008). Estilos relacionales parentales y ajuste adolescente. Infancia y Aprendizaje, 31(1), 93-106. http://dx.doi.org/10.1174/021037008783487093

Oliva, A., Parra, A., Sánchez-Queija, I. \& López, F. (2007). Estilos educativos materno y paterno: Evaluación y relación con el ajuste adolescente. Anales de Psicología, 23(1), 49-56.

Organización Internacional del Trabajo/Programa de Naciones Unidas para el Desarrollo (2009). Trabajo y familia: hacia nuevas formas de conciliación con corresponsabilidad social. Oficina Internacional del Trabajo/Programa de las Naciones Unidas para el Desarrollo. Santiago de Chile: OIT/PNUD.

Osorio de Rebellón, A., Rivas, S., Estévez, J. de I., Calatrava, M. \& López del Burgo, C. (2009). Evaluación de los estilos parentales en una muestra de estudiantes filipinos: implicaciones educativas. Revista Panamericana de Pedagogía, 14, 13-37.

Palacios, J. (1999). La familia y su papel en el desarrollo afectivo y social. En F. López, I. Etxebarría, M. J. Fuentes y M. J. Ortiz (Coords.), Desarrollo afectivo y social (pp. 267-284). Madrid: Pirámide.

Paredes, M. (2003). Los cambios en la familia en Uruguay: ¿hacia una segunda transición demográfica? En Unicef-Udelar, Nuevas formas de familia, perspectivas nacionales e internacionales (pp. 73-102). Uruguay: Unicef-Udelar.

Pardo, A. \& Ruiz, M. (2002). SPSS 11. Guia para el análisis de datos. Madrid: McGraw-Hill. 
Parra, A. \& Oliva, A. (2002). Comunicación y conflicto familiar durante la adolescencia. Anales de Psicología, 18(2), 215-231.

Parra, A., Oliva, A. \& Sánchez-Queija, I. (2004). Evolución y determinantes de la autoestima durante los años adolescentes. Anuario de Psicología, 35, 331-346.

Pastor, M. J. (2014). Estilos educativos percibidos en una muestra de niños de edad escolar en función de variables personales y familiares. Revista de Psicología Clinica con Niños y Adolescentes, 1(2), 133-139.

Patterson, G. R. (1982). Coercitive Family Process. Eugene, OR: Castalia. Patterson, G. R. (2002). The early developmental of coercitive family process. En J. B. Reid, G. R. Patterson y J. Snyder (Eds.), Antisocial behavior in children and adolescents: Developmental theories and models for intervention (pp. 25 44). Washington, DC: American Psychological Association.

Pérez Alonso-Geta, P. (2012). La socialización parental en padres españoles con hijos de 6 a 14 ańos. Psicothema, 24(3), 371-376.

Polo del Río, M. I., Fajardo, F., Martín, E., Gómez, T. \& León del Barco, B. (2012). Aceptación/rechazo parental en una muestra de adolescentes. Diferencias según sexo y edad. INFAD Revista de Psicología, 1(1), 329-336.

Prinzie, P., Onghena, P., Hellinckx, W., Grietens, H., Ghesquière, P. \& Colpin, H. (2004). Parents and Child Personality Characteristics as Predictors of Negative Discipline and Externalizing Problem Behavior in Children. European Journal of Personality, 18, 73-102. http://dx.doi.org/10.1002/per.501

Richaud de Minzi, M. C. (2005). Estilos parentales y estrategias de afrontamiento en niños. Revista Latinoamericana de Psicología, 37(1) 47-58.

Rodrigo, M. J., García, M., Máiquez, M. L. \& Triana, B. (2005). Discrepancias entre padres e hijos adolescentes en la frecuencia percibida e intensidad emocional en los conflictos familiares. Estudios de Psicología, 26(1), 21-34. 
Rodrigues, Y., Veiga, F., Fuentes, M. C. \& García, F. (2013). Parenting and Adolescents' Self-esteem: The Portuguese Context. Revista de Psicodidáctica, 18(2), 395-416. http://dx.doi.org/10.1387/ RevPsicodidact.6842

Rodríguez, M. A., Del Barrio, M. V. \& Carrasco, M. A. (2009). ¿Cómo perciben los hijos la crianza materna y paterna? Diferencias por edad y sexo. Escritos de Psicología, 2(2), 10-18.

Rodriguez, J., Pérez-Fuentes, M. del C. \& Gázquez, J. (2013). Relación entre el estilo educativo parental y el nivel de adaptación de menores en riesgo social. European Journal of Investigation in Health, Psychology and Education, 3(3), 301-318.

Ruvalcaba, N., Gallegos, J., Robles, F., Morales, A. \& González, N. (2012). Inteligencia emocional en la mejora de los estilos educativos de padres con hijos en edad escolar. Salud \& Sociedad, 3(3), 283-291.

Sabán, S., Herruzo, F. \& Raya, A. (2013). Relación entre estilos educativos familiares y la inclusión en Programas Diversificación Curricular: un elemento a considerar para la mejora de la convivencia escolar. Apuntes de Psicología, 31(2) 237-245.

Segura, M. \& Mesa, J. (2011). Enseñar a los hijos a convivir. Guía práctica para dinamizar escuelas de padres y abuelos. Bilbao: Desclée de Brouwer.

Simons, L. G. \& Conger, R. D. (2007). Linking mother-father differences in parenting to a typology of family parenting styles and adolescent outcomes. Journal of Family Issues, 28, 212-241. http://dx.doi.org/10.1177/0192513X06294593

Smetana, J. G. (1995). Parenting styles and conceptions of parental authority during adolescence. Child Development, 66(2), 299316. http://dx.doi.org/10.2307/1131579

Steinberg, L. (2001). We know some things: parent-adolescent relationships in retrospect and prospect. Journal of Research on Adolescence, 11, 1-19. http://dx.doi.org/10.1111/15327795.00001 
Tilano, L. M., Henao, G. C. \& Restrepo, J. A. (2009). Prácticas educativas familiares y desempeño académico en adolescentes escolarizados en el grado noveno de instituciones educativas oficiales del municipio de Envigado. El Ágora USB, 9(1) 35-51.

Torío, S., Peña, J. V. \& Inda, M. (2008). Estilos de educación familiar. Psicothema, 2(1), 62-70.

Torío, S., Peña, J.V. \& Rodriguez, M. del C. (2008). Estilos parentales. Revisión bibliográfica y reformulación teórica. Teoría Educativa 20, 151-178.

Tur-Porcar, A., Mestre, M. V., Samper, P. \& Malonda, E. (2012). Crianza y agresividad de los menores: ¿es diferente la influencia del padre y de la madre? Psicothema, 24(2), 284-288.

Valdivia, M. del C. (2007). Nuevo modelo de familia. En M. R. Buxarrais y M. P. Zeledón (Coords.), Las familias y la educación en valores democráticos. Retos y perspectivas actuales (pp. 35-70). Barcelona: Claret.

Valdivia, M. del C. (2010). Madres y padres competentes. Claves para optimizar las relaciones con nuestros hijos e hijas. España: Graó.

Vallejo, A., Osorno, R. \& Mazadiego, T. (2008). Estilos parentales y sintomatología depresiva en una muestra de adolescentes veracruzanos. Enseñanza e Investigación en Psicología, 13(1), 91-105.

Vidal, R. (2001) Conflicto psíquico y estructura familiar sistemas abiertos. Interacciones entre consciente e inconsciente, realidad psíquica y realidad, determinismo y azar. Montevideo: Psicolibros.

Viñas Poch, F., M. González Carrasco, Y. García Moreno, S. Malo Cerrato \& F. Casas Aznar (2015). Los estilos y estrategias de afrontamiento y su relación con el bienestar personal en una muestra de adolescentes. Anales de Psicología, 31(1), 226-2.

Recibido el 14 de agosto, 2015

Aceptado el 15 de abril, 2016 